\title{
An Increase of Maximal Intima-Media Thickness in Bilateral Carotid Arteries Strongly Suggests the Existence of Coronary Stenosis in Japanese Type 2 Diabetic Patients
}

\author{
Shimizu $\mathrm{H}^{1 *}$, Matsubara $\mathrm{F}^{1}$, Shiba $\mathrm{N}^{2}$, Takeda $\mathrm{M}^{2}$
}

Case Study

${ }^{1}$ Department of Diabetes and Endocrinology, International University of Health and Welfare (IUHW) Hospital, Nasushiobara, Japan.

${ }^{2}$ Department of Cardiology, International University of Health and Welfare (IUHW) Hospital, Nasushiobara, Japan.

\begin{abstract}
An association of an increase of maximal intima-media thickness (IMT) of carotid artery to coronary artery stenosis was evaluated in Japanese type 2 diabetic (T2DM) out-patients without any typical cardiac symptoms or history. Maximal IMT from common carotid artery to internal carotid artery was measured by ultrasonography in T2DM outpatients without any typical symptoms or history of coronary artery disease (CAD). The patients with a significant increase of maximal IMT over $1.5 \mathrm{~mm}$ were consulted to cardiologists, and the existence of coronary artery stenosis was evaluated by multiple detector-row computed tomography (MDCT). The patients with coronary artery stenosis suspected by MDCT were additionally evaluated the existence of CAD by coronary angiography. From April 2013 to December 2014, we found 17 patients with an increase of maximal IMT in bilateral carotid arteries and 9 patients with an increase of maximal IMT in unilateral carotid artery in T2DM outpatients. The patients with a significant increase of maximal IMT in bilateral carotid arteries had higher incidence of coronary artery stenosis by coronary angiography $(76.5 \%)$ than those in unilateral carotid artery $(11.1 \%)$. The $47.1 \%$ of the patients with an increase of maximal IMT in bilateral carotid arteries was judged to need percutaneous coronary intervention therapy. In conclusion, a significant increase of maximal IMT in bilateral carotid artery strongly suggests the existence of coronary artery stenosis in Japanese T2DM patients. Screening of maximal IMT in bilateral carotid arteries should be beneficial for the detection of silent ischemia in coronary artery.
\end{abstract}

Keywords: Intima-Media Thickness (IMT); Coronary Artery Stenosis; Type 2 Diabetes Mellitus.

\section{*Corresponding Author:}

Hiroyuki Shimizu, MD, PhD

Department of Diabetes and Endocrinology, International University of Health and WelfareHospital, Iguchi 537-3 Nasushiobara 329-2763 Japan. Tel: 81-287-37-2221, extn.: 2731

Fax: 81-287-39-3001

E-mail: hshimizu@iuhw.ac.jp

Recieved: February 18, 2015

Accepted: March 02, 2015

Published: March 03, 2015

Citation: Shimizu H, Matsubara F, Shiba N, Takeda M (2015) An Increase of Maximal Intima-Media Thickness in Bilateral Carotid Arteries Strongly Suggests the Existence of Coronary Stenosis in Japanese Type 2 Diabetic Patients. Int J Diabetol Vasc Dis Res, 3(2) 83-86. doi: http:// dx.doi.org/10.19070/2328-353X-1500016

Copyright: Shimizu $\mathbf{H}^{\circ}$ 2015. This is an open-access article distributed under the terms of the Creative Commons Attribution License, which permits unrestricted use, distribution and reproduction in any medium, provided the original author and source are credited.

\section{Introduction}

Asymptomatic coronary artery disease (CAD) and silent myocardial infarctions are common and sometimes lethalin diabetic subjects, and the incidence of CAD and asymptomatic myocardial infarction increases in diabetic patients [1]. However, it may be sometimes hard to prevent cardiac event in diabetic patients with diabetic autonomic neuropathy, because of no typical symptoms of ischemic heart disease [2]. Effective and non-invasive methods which screen the existence of silent ischemic heart disease in T2DM patients have not been established enough yet. To screen ischemic heart disease by coronary angiography in all diabetic patients is so invasive and in appropriate, because the burden to the body is so heavy in T2DM patients who may have renal damage by hyperglycemia. Ultrasonography is easy to be done in the clinics for the screening method, and not invasive to the patients, even if they had renal damage. Carotid artery intima-media thickness (IMT) measurement by ultrasonography has been reported to be correlated with coronary artery stenosis [3]. Carotid maximum IMT is useful for screening asymptomatic type 2 diabetic patients with severe CAD equivalent to revascularization [4]. However, an association of maximal IMT to silent ischemia in T2DM patients has not yet been reported enough. In our hospital, we are screening the existence of carotid artery stenosis by ultrasonography in type 2 diabetic (T2DM) outpatients, and the patients with a significant increase of maximal IMT in carotid artery are consulted to cardiologists to screen the existence of ischemic heart disease in order to prevent silent myocardial infarction. We summarized the data about an association of an increase of maximal IMT to coronary artery stenosis in T2DM out-patients without any typical symptoms or past history of CAD in our hospital.

\section{Subjects and Design}

T2DM out-patients without any typical symptomsor history of 
ischemic heart disease had been screened the existence of arteriosclerosis by measuring intima-media thickness (IMT) by ultrasonography in carotid artery from April 2013 to December 2014. We evaluated maximal IMT in bilateral carotid arteriesof the patientsby using ultrasonography (ALOKA Prosound $\alpha 7$, Hitachi Aloka Medical, Ltd., Tokyo, Japan). Maximal IMT was obtained from the observation from common carotid artery to internal carotid artery. Maximal IMT includes plaque thickness in any segment of carotid artery. It is recommended that patients with more than $1.1 \mathrm{~mm}$ mean IMT at the carotid artery and/or more than 20 years duration of diabetes should be screened for CAD by carrying out MDCT [5]. We are using maximal IMT $>1.5 \mathrm{~mm}$ or carotid artery stenosis over $50 \%$ as the cut-off point of carotid arteriosclerosis according to the previous report [6]. After obtaining the informed consent from each patient, the patients with a significant increase of maximal IMT were consulted to cardiologists in our hospital, and the existence of coronary artery stenosis was investigated.

The existence of coronary artery stenosis was screened in the department of cardiology by multiple detector-row computed tomography (MDCT) in the patients with diabetic nephropathy from stage 1 to stage 3. According to the results of MDCT, the indication of coronary angiography (CAG) was decided by cardiologists in the patients who were suspected to have any coronary artery sclerosis. However, coronary angiography was first selected to evaluate coronary artery stenosis in the patients with diabetic nephropathy at stage 4 , to avoid the development of further renal damage by using contrast medium on MDCT.

The existence of coronary stenosis was finally diagnosed by cardiologists from the results obtained by CAG in the patients who may have coronary artery stenosis suspected by MDCT, and the necessity of percutaneous coronary intervention (PCI) therapy was determined according tothe results of CAG.

Pulse wave velocity (PWV), ankle-brachial index (ABI), and toe-brachial index (TBI) measurement
PWV, BI, and TBI were measured with the patient in a supine position on a bed by using the Forum ABI/PWV (Omron Colin Co., Ltd., Tokyo, Japan) [7]. ABI was calculated by dividing calf systolic blood pressure by brachial systolic blood pressure, and TBI was calculated by dividing big toe systolic blood pressure by brachial systolic blood pressure.

\section{Statistical Analysis}

All data are expressed as mean \pm SD. The statistical analysis was performed by the analysis of variance, followed by Student's t-test for the individual comparison of the means. The difference of the incidence of coronary artery stenos is between two groups was performed by $\lambda^{2}$-test.

\section{Results}

From April 2013 to December 2014, we have experienced 26 outpatients with significant increases in maximal IMT in unilateral or bilateral carotid arteries by ulrasonographic observation. We analyzed the results of the following investigations by MDCT and CAG by dividing those patients into 2 groups: one is showing an increase of maximal IMT in a single carotid artery (one-side, right or left; unilateral group), and another is in both carotid arteries (bilateral group). Nine patients showed an increase of maximal IMT in unilateral carotid artery, and 17 patients showed an increase of maximal IMT in bilateral carotid arteries. The averages of maximal IMT in unilateral and bilateral carotid arteries were $2.26 \pm 0.67 \mathrm{~mm}, 2.87 \pm 0.94 \mathrm{~mm}$, respectively, and there was no significant difference between both groups.

Clinical backgrounds and recent glycemic control status of those patients were shown in Table 1 . There were no significant differences in clinical backgrounds, and recent laboratory data between the patients with an increase of maximal IMT in unilateral and bilateral carotid arteries. One patient in each of unilateral and bilateral groups $(11.1 \%, 5.9 \%$, respectively) showed the reduction of ABI below 0.9 , and one patient in unilateral group (11.1\%), two

Table 1. Clinical background of the patients.

\begin{tabular}{|l|l|l|}
\hline & \multicolumn{1}{|c|}{ Unilateral } & \multicolumn{1}{c|}{ bilateral } \\
\hline Number (male / female) & $9(6 / 3)$ & $17(15 / 2)$ \\
\hline Age (year-old) & $63.0 \pm 8.4$ & $66.2 \pm 9.8$ \\
\hline Body Mass Index (kg/m²) & $26.2 \pm 4.8$ & $24.6 \pm 4.9$ \\
\hline Duration of diabetes (year) & $10.2 \pm 8.0$ & $15.8 \pm 13.6$ \\
\hline Treatment (none/OHA/insulin) & $2 / 3 / 4$ & $3 / 5 / 9$ \\
\hline Diabetic Retinopathy (none/simple/proliferative/NA) & $6 / 2 / 1$ & $8 / 5 / 3 / 1$ \\
\hline Diabetic nephropathy (stage 1/2/3/4/NA) & $2 / 5 / 1 / 1$ & $1 / 8 / 4 / 1 / 3$ \\
\hline Blood glucose (mg/dl) & $239.4 \pm 92.0$ & $209.6 \pm 119.3$ \\
\hline HbA1c $(\%)$ & $8.72 \pm 2.81$ & $8.19 \pm 1.59$ \\
\hline LDL-cholesterol (mg/dl) & $122.7 \pm 20.7$ & $115.5 \pm 29.8$ \\
\hline HDL-cholesterol (mg/dl) & $51.6 \pm 13.8$ & $52.7 \pm 11.3$ \\
\hline Triglyceride (mg/dl) & $222.9 \pm 198.4$ & $120.4 \pm 71.1$ \\
\hline baPWV (cm/second) & $1731.0 \pm 246.7$ & $1838.2 \pm 380.6$ \\
\hline ABI & $1.14 \pm 0.06$ & $1.15 \pm 0.15$ \\
\hline TBI & $0.79 \pm 0.15$ & $0.84 \pm 0.15$ \\
\hline
\end{tabular}

NA: not assessed, baPWV: brachial-ankle pulse wave velocity 
patients in bilateral group $(11.8 \%)$ showed the reduction of TBI below 0.6, indicating the existence of obliterating arteriosclerosis. Table 2 summarized the results of MDCT and CAG. In three patients showing an increase of maximal IMT in unilateral carotid artery, coronary artery stenosis was suspected by MDCT. However, a significant coronary artery stenosis over $50 \%$ was found by CAG in only one patient who was judged to need PCI (11.1 $\%)$. In contrast, coronary artery stenosis was suspected by MDCT in 14 patients with an increase of maximal IMT in bilateral carotid arteries. One patient did not accept CAG, and 13 patients received CAG. A significant coronary artery stenosisover $50 \%$ was foundby CAG in all 13 patients with the increase of maximal IMT in both carotid arteries $(76.5 \%$ of all patients). The percentage of the patients with a significant coronary artery stenosis was significantly $(\mathrm{p}<0.005)$ higher in those patients than unilateral group. As a consequence of CAG, $47.1 \%$ of total patients with an increase of maximal IMT in bilateral carotid arteries was judged to need PCI. In contrast, only one patient was judged to need PCI in unilateral group. The incidence of patients who were judged to need PCI tended to be higher in bilateral group than unilateral group, but the difference was not statistically significant because of a small number of patients.

\section{Discussion}

It has been recently reported that max IMT in the $\geq 50 \%$ stenosis group by multi-slice computed tomography coronary angiography estimation was significantly greater than the $0-25$ and $25-50 \%$ stenosis group [8]. In type 2 diabetic patients without apparent cardiovascular disease, the addition of max-IMT to conventional risk factors substantially improves the risk stratification for CAD [9]. However, within our knowledge, the association of the difference in an increase of maximal IMT between unilateral and bilateralcarotid arteries to coronary artery stenosis has not been investigated by now. In our hospital, the patients with an increase of maximal IMT over $1.5 \mathrm{~mm}$, who have no typical cardiac symptoms or history of CAD, are screened by cardiologists by using MDCT. AS a consequence, we found the obvious difference of coronary artery stenosis in the increases of maximal IMT between unilateral and bilateral carotid arteries, independently of the recent laboratory data about metabolic factors. PCI was indispensable in $47.1 \%$ of the patients in bilateral group to avoid future cardiac event. The present data obtained herein strongly indicate that Japanese T2DM patients with a significant increase of maximal IMT in bilateral carotid arteries over $1.5 \mathrm{~mm}$ may have high incidence of silent CAD, and such patients should be consulted to cardiologists as soon as possible, even if they have not had any symptoms of ischemic heart disease by now, and to be screened about the existence of coronary artery stenosis. Therefore, those patients could receive preventive PCI to avoidcardiac event, if necessary by the evaluation of CAG results.

The reason why the patients with an increase of maximal IMT in bilateral coronary arteries showed higher incidence of coronary

Table 2. Incidence of patients with coronary stenosis and needed PCI.

\begin{tabular}{|l|c|c|c|}
\hline & total & unilateral & bilateral \\
\hline Number of patients (male/female) & $26(21 / 5)$ & $9(6 / 3)$ & $17(15 / 2)$ \\
\hline $\begin{array}{l}\text { Number of patients with coronary artery stenosis } \\
\text { suspected by CT image }\end{array}$ & 17 & $\begin{array}{c}3 / 9 \\
(33.3 \%)\end{array}$ & $\begin{array}{c}14 / 17 \\
\left(82.4 \%^{*}\right)\end{array}$ \\
\hline $\begin{array}{l}\text { Number of patients with coronary artery stenosis } \\
\text { diagnosed by CAG }\end{array}$ & 14 & $\begin{array}{c}1 / 9 \\
(11.1 \%)\end{array}$ & $\begin{array}{c}13 / 17 \\
(76.5 \% * *)\end{array}$ \\
\hline Number of patients judged to need PCI & 9 & $\begin{array}{c}1 / 9 \\
(11.1 \%)\end{array}$ & $\begin{array}{c}8 / 17 \\
(47.1 \%)\end{array}$ \\
\hline \multicolumn{2}{|c|}{$(*: \mathrm{p}<0.05, * *: \mathrm{p}<0.005)$} \\
\hline
\end{tabular}

artery stenosis than those in only unilateral coronary artery was not clarified at all in this analysis, because there were no clear differences in the recent data about various metabolic factors about diabetes and lipid metabolism. There may be a possibility that an increase of maximal IMT in bilateral carotid arteries may suggest the development of arteriosclerosis in broad segment of arteries throughout the whole body, and arteriosclerosis may develop segmentally or partially in the patients with an increase of maximal IMT only in unilateral carotid artery. However, because of the small number of patients, several factors could not be considered enough in the present analysis, the results obtained herein is limited. Large replication studies should be necessary to finally conclude the present hypothesis.

\section{Conclusion}

In conclusion, an increase of maximal IMT in bilateral carotid arteries strongly suggests the existence of coronary artery stenosisin T2DM patients, independently of recent metabolic status. Screening of maximal IMT in bilateral carotid arteries should be beneficial for the detection of silent CAD in T2DM patients.

\section{References}

[1]. Airaksinen KE (2001) Silent coronary artery disease in diabetes-a feature of autonomic neuropathy or accelerated atherosclerosis? Diabetologia 44: 259-266.

[2]. Hume L, Oakley GD, Boulton AJ, Hardisty C, Ward JD (1986) Asymptomatic myocardial ischemia in diabetics and its relationship to diabetic neuropathy: an exercise electrocardiography study in middle-aged diabetic men. Diabetes Care 9: 384-388.

[3]. Kitagawa K, Hougaku H, Yamagami H, Hashimoto H, Itoh T, et al. (2007) OSACA2 Study Group. Carotid intima-media thickness and risk of cardiovascular events in high-risk patients. Results of the Osaka Follow-Up Study for Carotid Atherosclerosis 2 (OSACA2 Study). Cerebrovasc Dis 24: 35-42

[4]. Irie Y, Katakami N, Kaneto H, Nishio M, Kasami R, et al. (2013) The utility of carotid ultrasonography in identifying severe coronary artery disease in asymptomatic type 2 diabetic patients without history of coronary artery disease. Diabetes Care 36: 1327-1334.

[5]. Nair SB, Malik R, Khattar RS (2012) Carotid intima-media thickness: ultrasound measurement, prognostic value and role in clinical practice. Postgrad Med J 88: 694-699.

[6]. Nishioka H, Furukawa N, Shimoda S, Nishida K, Nakaura T, et al. (2010) Predictors of coronary heart disease in Japanese patients with type 2 diabetes: Screening for coronary artery stenosis using multudetector computed tomography. J Diabetes Invest 22: 50-55. 
[7]. Shimizu H, Shimomura K, Negishi M, Oh-I S, Tomita Y, et al. (2004) Glycaemic control and the increase in pulse wave velocity (PWV) in type 2 diabetic patients. Diabet Med. 21: 804-805.

[8]. Kasami R, Kaneto H, Katakami N, Sumitsuji S, Yamasaki K, et al. (2011) Relationship between carotid intima-media thickness and the presence and extent of coronary stenosis in type 2 diabetic patients with carotid athero- sclerosis but withough history of coronary artery disease. Diabetes Care 34: 468-470.

[9]. Irie Y, Katakami N, Kaneto H, Kasami R, Sumitsuji S, et al. (2012) Maximum carotid intima-media thickness improves the prediction ability of coronary artery stenosis in type 2 diabetic patients without history of coronary artery disease. Atherosclerosis 221: 438-444. 